\title{
Current progress in non-surgical embryo transfer with fresh and vitrified/warmed pig embryos
}

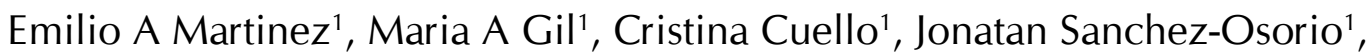 \\ Jesus Gomis ${ }^{1}$, Inmaculada Parrilla ${ }^{1}$, Miguel A Angel ${ }^{1}$, Heriberto Rodriguez- \\ Martinez ${ }^{2}$, Xiomara Lucas ${ }^{1}$, Jose L Vazquez ${ }^{1}$, Juan M Vazquez ${ }^{1}$ \\ and Jordi Roca ${ }^{1}$
}

${ }^{1}$ Department of Animal Medicine and Surgery, Murcia University, Campus de Espinardo, 30100 Murcia, Spain, ${ }^{2}$ Department of Clinical and Experimental Medicine, Linköping University, SE-581 85 Linköping, Sweden

Embryo transfer (ET) should play a critical role in the pig industry because it allows the movement and introduction of new genetic material into a herd with minimal risk of disease transmission and reduced transportation costs. In addition, embryo movement could prevent the potential health and welfare problems associated with transporting live pigs. Although the first successful ET was reported more than 60 years ago, the commercial use of this procedure in pigs is still in its infancy. The surgical requirements for embryo collection and transfer and the difficulties with embryo cryopreservation have prevented its use in pigs, unlike other livestock. However, new methodologies have been developed in the past decade to enable successful non-surgical ET and embryo cryopreservation that could allow the commercial use of ET in the pig industry. This review focuses on the development of these technologies with emphasis on our own findings. Specifically, we discuss the basic aspects of a non-surgical deep-uterine ET procedure and describe several factors that affect its efficacy in the transfer of fresh and short-term cultured embryos. Finally, we conclude with a brief discussion on the use of this procedure with long-term stored embryos.

\section{Introduction}

Little information is available concerning the reproductive performance of recipients after surgical transfers of fresh embryos. Most of the reports described experimental studies evaluating the pregnancy rates and fetus numbers 30-35 days after transfer. In general, these studies reported pregnancy rates of 60-80\% with 5-8 fetuses per pregnant recipient (Polge 1982, Blum-Reckow \& Holtz 1991, Wallenhorst \& Holtz 1999). In only a limited number of surgical ET studies were the recipients allowed to carry litters to term, achieving farrowing rates of $50-80 \%$ and litter sizes of 6-8 piglets (James et al. 1980, Cameron et al. 1989, Niemann et al. 1989). Although Polge and Day (1968) demonstrated that pregnancy could be established in pigs through non-surgical $\mathrm{ET}$, the procedure was considered an inefficient technique for many years due to the complex anatomy of the porcine cervix and uterus. However, new non-surgical procedures for embryo deposition were developed in the 1990s, achieving farrowing rates of $5-41 \%$ and litter sizes of 5-7.5 piglets (reviewed in Cameron et al. 2006). Among these procedures, the most promising involved the placement of embryos into the uterine body of non-sedated sows (Hazeleger \& 
Kemp 1994). Although the farrowing rates (42\%) and the litter sizes (7.5 piglets) reported using that procedure (Ducro-Steverink et al. 2004) were comparable to those achieved with surgical transfers, improvements were still needed to increase the reproductive performance of the recipients after non-surgical ET. From physiological and practical perspectives, three limitations of non-surgical uterine body ET procedure could be considered: the place of the deposition of the embryos, the embryo developmental stage at transfer and the type of recipients. Under natural conditions, after entry into the uterus, pig embryos remain near the tip of the uterine horn until day 6-7 (day 0 = onset of estrus), progressing then towards the uterine body (Dziuk, 1985). For ET purposes, embryos are usually collected from the tip of the uterine horns at morula and blastocyst stages on days 5 and 6 , respectively. Therefore, it appears to be reasonable that the transfer of these embryos to anterior portions of the uterine horns of the recipients might have some advantages compared with the transfer into the uterine body. In agreement with this hypothesis, results from surgical ET indicated that the uterine body is a less appropriate place than the middle or anterior quarter of the uterine horn (Wallenhorst and Holtz, 1999). The stage of the embryos at transfer seems to be other limiting factor of the uterine body non-surgical ET procedure since pregnancy rates fell dramatically with transfers of embryos at the morula stage in comparison with those achieved using blastocysts (Hazeleger and Kemp, 1994). The third limitation of that procedure is that only sows can be used as recipients because the cervix of gilts is more tightly closed and does not permit the penetration of the uterine-body catheter (Hazeleger, 1999). To overcome these limitations, we designed a research program directed toward developing a new procedure for the non-surgical transfer of porcine embryos deep into a uterine horn of non-sedated gilts and sows. The preliminary results of this method were published at the 2001 International Conference on Pig Reproduction (Martinez et al. 2001), and although the procedure will continue to be improved in the next years, the results already achieved with fresh and vitrified embryos represent a fundamental advance in the widespread commercial use of ET in pigs.

\section{Safety and effectiveness of the non-surgical deep-uterine (NsDU) embryo transfer procedure}

In 1999, we designed an NsDU ET catheter to avoid the two principal obstacles in the swine genital tract, the cervical folds and the length and coiled nature of the uterine horn (Martinez et al. 2001). The catheter, currently produced by Minitube (Tiefenbach, Germany), has an adequate propulsion force to pass through the cervix and sufficient flexibility to progress along the uterine horn. Intrauterine insertions of the catheter are performed in regular insemination crates at days 4-6 of the estrous cycle in non-sedated gilts and sows. The efficacy of the insertions is provided in Table 1 . In $90 \%$ of the gilts and sows, the catheter can be correctly inserted into the second or third quarter of the uterine horn (Figure 1) in less than 5 min. Although the number of incorrect insertions is low, the ability to ascertain the precise location of the catheter during the insertion is important to maximize the efficacy of the procedure. More than $95 \%$ of the transfers are correctly predicted, minimizing the embryonic waste due to incorrect transfer that results in poor pregnancy rates. The NsDU ET procedure is simple; however, acquiring the skills necessary to perform this new procedure requires training. The minimum number of cases required for proficiency must be determined because it impacts the outcome of the procedure. Figure 2 shows the results obtained from NsDU transfers with fresh embryos by the same operator over time. These data indicate that NsDU transfer skills improve with practice, leading to an increase in the farrowing rates and a decrease in the duration of the catheter insertions. Therefore, completion of a training period should be mandatory when 
Table 1 Efficacy of the insertions of non-surgical deep-uterine catheters in gilts and sows at days 4-6 of the estrous cycle (adapted from Martinez et al. 2004 and Cuello et al. 2005).

\begin{tabular}{|c|c|c|c|}
\hline \multirow[t]{2}{*}{ Parameter } & \multicolumn{2}{|c|}{ Type of recipient } & \multirow[t]{2}{*}{ Observations } \\
\hline & Gilts* & Weaning sows & \\
\hline Possibility of insertions (\%) & $85-90$ & 95 & \\
\hline Correct insertions $(\%)^{* *}$ & $80-90$ & $90-93$ & \\
\hline Correct prediction of insertions (\%) & $>95$ & $>95$ & \\
\hline $\begin{array}{l}\text { Depth of the insertions into a uterine horn (anterior } \\
\text { quarters) }\end{array}$ & $2 \mathrm{nd}-3 \mathrm{rd}$ & $2 \mathrm{nd}-3 \mathrm{rd}$ & In $90 \%$ of recipients \\
\hline Non-difficulties during insertions (\%) & $>70$ & $>80$ & \\
\hline Day of the recipient cycle ${ }^{* * *}$ & $4-6$ & $4-6$ & \\
\hline Time required for insertions (min) & $2-5$ & $2-3$ & \\
\hline $\begin{array}{l}\text { Good (no reaction) behavior of the females during the } \\
\text { procedure }(\%)\end{array}$ & $>90$ & $>90$ & $\begin{array}{l}\text { Worse in gilts than in } \\
\text { sows }\end{array}$ \\
\hline Perforations & No & No & \\
\hline Infections after ET & No & No & \\
\hline
\end{tabular}

*With more than two previous estrus cycles. **As determined by laparotomy or after slaughter (gilts) and by radiography or by the shape of the catheter after removal, whether it was straight or bent (sows). ${ }^{* *}$ Day $0=$ onset of estrus.
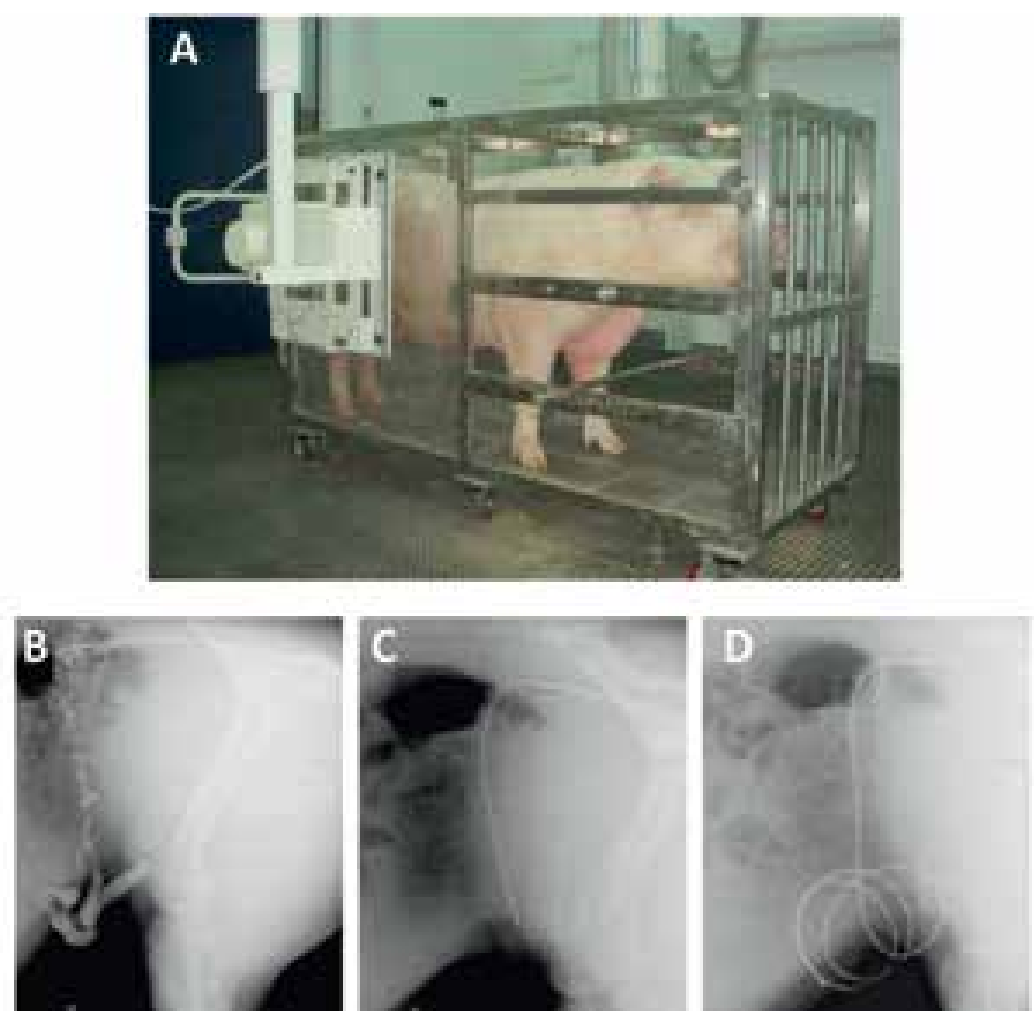

Figure 1 Radiographic images obtained in a non-sedated sow (A) after introduction of contrast liquid (B): the cervical canal, the uterine body, the uterine bifurcation and the beginning of the uterine horns are clearly visible. (C) The insertion of the non-surgical deep-uterine catheter into the uterine body and (D) its progression along a uterine horn. 


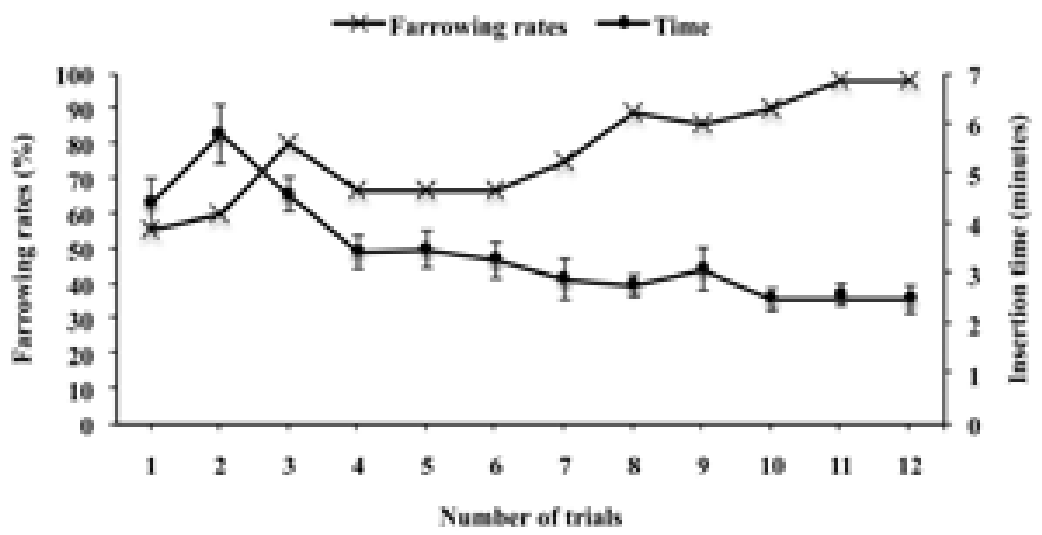

Figure 2 Farrowing rates and duration of the non-surgical deep uterine catheter insertions during the training curve. Each trial was conducted in separate sessions over a 3-year period and consisted of $7-11$ embryo transfers $(n=111)$ by the same operator. Trials are indicated in chronological order from older (trial 1) to most recent (trial 12).

initiating an NsDU ET program in either research or commercial contexts. Animal welfare is another important factor to consider. As shown in Table 1, more than $90 \%$ of the females react well to the catheter insertions, suggesting that the procedure is well tolerated and painless even when insertions are problematic. The absence of uterine infections following transfers is also remarkable given the potential risk for infection during non-surgical ET with the manipulation of the uterine environment when the animal is under the influence of progesterone (De Winter et al. 1996). With more than $160 \mathrm{NsDU}$ ETs performed by a private company (Selección Batallé SA, Girona, Spain) during the last 3 years, we can affirm that uterine infections can be completely avoided by using a clean ET room and sterile instruments, carefully cleansing and disinfecting the perineal area and administering an antibiotic on the day of the ET. On the other hand, although it has not been adequately evaluated, the potential injury or damage to the sow's reproductive tract due to the insertion of the NsDU ET catheter appears to be minimal. The absence of bleeding during and after catheter insertions and the excellent fertility results obtained after transfers support that statement.

\section{Non-surgical deep-uterine transfer with fresh embryos}

Early research on surgical and non-surgical ETs in pigs has been reviewed in detail elsewhere and is not discussed herein (Hazeleger \& Kemp 2001, Cameron et al. 2006). Instead, we discuss our own research findings and describe several factors that affect the success rate of NsDU ET of fresh embryos.

\section{Superovulation of the donors}

Although the ovulation rates differ among pig breeds, lines, and animals within a breed (Caárdenas \& Pope 2002), 15-25 oocytes could be proposed as an average for this species. Successful surgical and non-surgical ET requires 15-23 (Polge 1982, Cameron et al. 1989) and 24-30 (Hazeleger et al. 2000a, Martinez et al. 2004) embryos per transfer, respectively, with a donor:recipient ratio of approximately $1: 1$. However, in practice, the situation is different and can be affected by several factors, such as pregnancy rate (not all donors become pregnant after insemination), fertilization rate (not all oocytes are fertilized), stage of embryonic development at 
time of collection (embryos are not always at the optimal stage of development when collected), embryo quality (not all embryos collected are transferable), and the recovery rate (some embryos are lost during collection). Therefore, the donor:recipient ratio should be increased to approximately $2: 1$. To reduce this proportion, the donors can be superovulated with equine chorionic gonadotropin (eCG) (reviewed in Brüssow et al. 2009); however, several problems have been associated with this treatment, including an inconsistent ovulatory response (Furstoss et al. 2012), high percentages of unfertilized oocytes, poor embryo quality (Holtz \& Schlieper 1991) and embryonic mortality (Guthrie et al. 1974). At contrast, Hazeleger et al. (2000a) reported an increased number of transferable embryos by using a high dosage of eCG (1500 IU) in comparison to that achieved with a dosage of $1000 \mathrm{IU}$ eCG. The higher eCG dose tended to give lower pregnancy rates, but did not affect the number of high-quality embryos. We recently developed an NsDU ET program in purebred Duroc sows, a breed that is convenient for superovulation due to its low ovulation rate. We evaluated the aforementioned doses of eCG (1000 and 1500 IU), administered $24 \mathrm{~h}$ post-weaning. The sows in estrus 48-72 $\mathrm{h}$ after eCG dosing were treated with $750 \mathrm{IU}$ human chorionic gonadotropin, inseminated 12 and $24 \mathrm{~h}$ after the onset of estrus, and used as embryo donors. Untreated sows with natural post-weaning estrus were used as the controls. Embryos were collected 5-6 days after the onset of estrus, morphologically evaluated, and non-surgically transferred deep into a uterine horn of the recipients (30 embryos per transfer). All donors were pregnant at the time of laparotomy, except two sows (8.3\%) from the higher eCG-dose group. Both of these sows displayed polycystic ovaries and no signs of ovulation. The number of corpora lutea and embryos increased with the higher eCG dose (Figure 3). Contrary to the earlier reports, we did not observe any effect of the treatments on either the number of unfertilized oocytes or the embryo quality. The results from the NsDU ET (Figure 4) indicated no differences between the control and treated groups in the pregnancy and farrowing rates or in the litter sizes. These results indicate that in purebred Duroc sows the number of donors per recipient can be decreased from 2.1 to 1.5 through superovulation, and that $1000 \mathrm{IU}$ eCG is adequate to obtain an acceptable number of transferable embryos and satisfactory reproductive performance of the recipients. Nevertheless, these data should not be extrapolated to other breeds or lines in which the superovulatory response could differ. In Danish DanBred crossbred (Landrace x LargeWhite) sows, for example, a dose of 1000 IU eCG increased the number of corpora lutea and transferable embryos approximately 1.6 times over that of the corresponding controls, resulting in a donor:recipient ratio of approximately to 1:1.2 (with 30 embryos transferred per recipient).

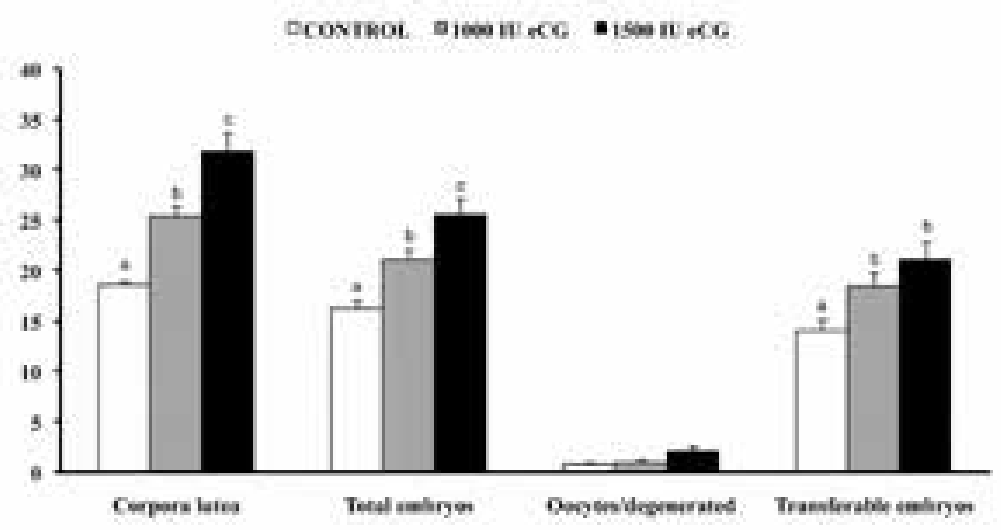

Figure 3 Number of corpora lutea, total embryos, oocytes/degenerated embryos and transferable embryos obtained from superovulated purebred Duroc sows $(\mathrm{n}=78)$ using different dosages of eCG (mean \pm SEM). ${ }^{a, b, c l n}$ the same parameter $\mathrm{P}<0.05$. 

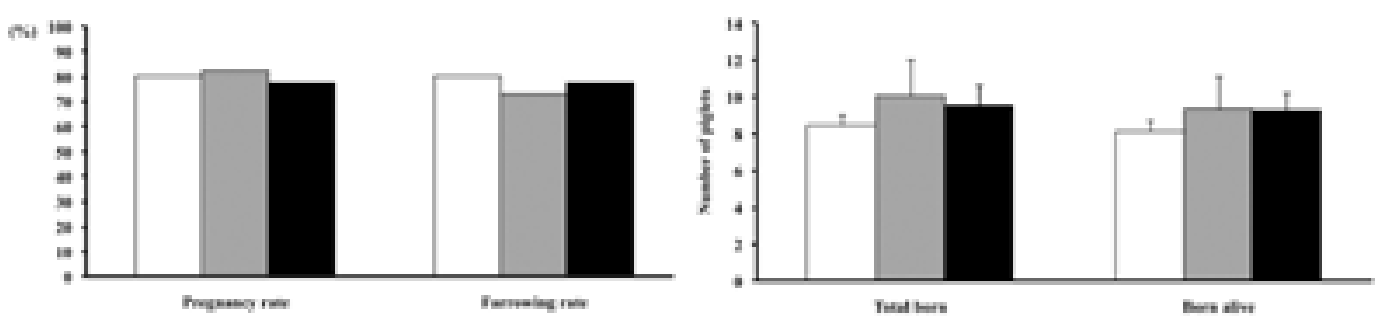

Figure 4 Fertility $(\%)$ and prolificacy (mean \pm SEM) of the recipients $(n=34)$ after non-surgical deep-uterine transfers of embryos collected from control (white bars) and superovulated purebred Duroc sows with 1000 IU (grey bars) and 1500 IU (black bars) eCG.

Degree of synchrony between the stage of embryo development and the recipients

Several trials involving surgical ET clearly demonstrated that higher pregnancy rates could be achieved when the transfers are performed on recipients that started estrus after the donors than those achieved using recipients that were in estrus before the donors (Polge 1982, Wilde et al. 1988, Blum-Reckow \& Holtz 1991). In contrast, studies involving non-surgical ET into the uterine body indicated that transfers to recipients ovulating 18-36 $\mathrm{h}$ after the donors resulted in lower pregnancy rates than those performed on recipients ovulating from $24 \mathrm{~h}$ before to 12 $\mathrm{h}$ after the donors (Hazeleger et al. 2000b). Our results with NsDU ET mirror those achieved in the surgical experiments, not those obtained with the non-surgical uterine body ETs. In our experiments (Tables 2 and 3), the pregnancy rates decreased significantly if the estrus of

Table 2 Effects of the varying degrees of synchrony between the recipients and donors on the farrowing rates and litter sizes after non-surgical deep-uterine transfers of 30 fresh porcine embryos at the morula and/or unhatched blastocyst stages.

\begin{tabular}{lcccc}
\hline & \multicolumn{4}{c}{ Synchrony recipients-donors (h)* } \\
\cline { 2 - 5 } & -24 & 0 & +24 & +48 \\
\hline Recipients $(\mathrm{n})$ & 8 & 26 & 33 & 12 \\
Pregnancy $(\mathrm{n} / \%)$ & $1(12.5)^{\mathrm{a}}$ & $16(61.5)^{\mathrm{b}}$ & $29(87.9)^{\mathrm{c}}$ & $5(41.7)^{\mathrm{ab}}$ \\
Farrowing $(\mathrm{n} / \%)$ & - & $16(61.5)^{\mathrm{ab}}$ & $27(81.8)^{\mathrm{b}}$ & $5(41.7)^{\mathrm{a}}$ \\
Total born (mean $\pm \mathrm{SEM})$ & - & $10.4 \pm 0.8$ & $9.6 \pm 0.7$ & $9.2 \pm 1.4$ \\
Born alive (mean \pm SEM) & - & $10.1 \pm 0.7$ & $9.1 \pm 0.6$ & $8.6 \pm 1.3$ \\
\hline
\end{tabular}

*Recipients in estrus before $(-)$ or after $(+)$ donors. ${ }^{a, b, c,}$ Different superscripts within the same row differ $(\mathrm{P}<$ 0.05).

Table 3 Reproductive parameters of the recipients in estrus $24 \mathrm{~h}$ later than the donors after non-surgical deeputerine transfer of 30 fresh porcine embryos.

\begin{tabular}{lcc}
\hline & \multicolumn{2}{c}{ Embryonic stage* } \\
\cline { 2 - 3 } & Morula & Blastocyst \\
\hline No. of recipients & 21 & 12 \\
Pregnancy rate $(\mathrm{n} / \%)$ & $19(90.5)$ & $10(83.3)$ \\
Farrowing rate $(\mathrm{n} / \%)$ & $17(81.0)$ & $10(83.3)$ \\
Total born (mean \pm SEM) & $9.9 \pm 0.8$ & $9.2 \pm 0.7$ \\
Born alive (mean \pm SEM) & $9.2 \pm 0.8$ & $9.0 \pm 0.8$ \\
\hline
\end{tabular}

Embryos at the morula and unhatched blastocyst stages were collected from donors at days 5 and 6 (day $0=$ onset of estrus), respectively, and were transferred to the recipients on days 4 and 5, respectively. 
recipients began $24 \mathrm{~h}$ before $(-24 \mathrm{~h})$ that of the donors. The highest pregnancy rates were achieved when the recipients were $+24 \mathrm{~h}$ asynchronous with the donors (87.9\%), regardless of the embryonic stage (day 5 morulae or day 6 blastocysts) used for the transfers. A recipient asynchrony of $+48 \mathrm{~h}$ was adequate for the blastocysts but not for the morulae transfers, most likely because in the latter case, the recipients were on the third day of the cycle and some still exhibited signs of estrus. The discrepancies among the studies using surgical and non-surgical ET could be related to the site of embryo placement in each procedure; therefore, additional data are needed to support the assertion that transferred embryos prefer a "younger" environment.

\section{Short-term (24 h) storage of fresh embryos}

From a commercial standpoint, the embryos must be transported from the donor to the recipient farms requiring a storage period. In vitro culture can be used as a method for short-term embryo storage. High blastocyst formation rates $(96 \%)$ were noted when in vivo derived 2- to 4-cell embryos were cultured in vitro for 3-4 days (Cuello et al. 2007). Although these blastocysts had lower cell numbers compared with their in vivo counterparts, no difference was noted in the in vivo developmental ability after surgical ET between the two types of blastocysts (BlumReckow \& Holtz 1991, Macháty et al. 1998). Because this storage method has the potential to maintain embryo viability during transport to the recipient farms, additional research is needed to evaluate the usability of these embryos for NsDU ET and to establish optimum culture conditions.

In some cases, a period of $24 \mathrm{~h}$ between the collection and transfer can enable the longdistance transportation of the embryos to their recipients. Thus, using surgical ET, acceptable farrowing rates (50-60\%) and litter sizes (5-8 piglets) were obtained after a transoceanic transport of the embryos (James et al. 1980, Niemann et al. 1989). More recently, Rubio Pomar et al. (2004) investigated different incubation temperatures and culture media and concluded that in vivo-derived early blastocysts stored at $25^{\circ} \mathrm{C}$ in a serum-free BSA medium for $24 \mathrm{~h}$ are at a suitable developmental stage for ET and provide high-quality embryos. Unfortunately, these results were not confirmed by the transfer of the stored embryos. In a recent study, we tested two types of culture media that do not require controlled $\mathrm{CO}_{2}$ gassing (NCSU23-HEPES-BSA and TL-HEPES-PVA) and two temperatures (25 and $\left.37{ }^{\circ} \mathrm{C}\right)$ for a 24-h in vitro culture of in vivo-derived morulae and early blastocysts. Embryos cultured in NCSU23-BSA-fetal calf serum medium at $38.5^{\circ} \mathrm{C}$ in an atmosphere of $5 \% \mathrm{CO}_{2}$ in air were used as a control. Our results indicated that the survival rates were negatively affected at 25 ${ }^{\circ} \mathrm{C}$ when the chemically defined medium (TL-HEPES-PVA) was used (Table 4). The reasons for the discrepancy between our results and those of Rubio Pomar et al. (2004) are unclear, although probably relate to the presence of BSA in the culture medium used in that study. In our experiment, no differences in the survival rates were detected in the embryos cultured at $37^{\circ} \mathrm{C}$, except for a developmental delay at $24 \mathrm{~h}$ in all groups compared with the control. This developmental delay was more profound when the embryos were incubated at $25{ }^{\circ} \mathrm{C}$. Unlike the controls, none of the embryos cultured in the experimental media at $37{ }^{\circ} \mathrm{C}$ hatched at the end of culture period. Because no two batches of serum or serum components are alike, and because their use carries a risk of disease, we selected the chemically defined medium to evaluate the effects of the embryo culture at $37^{\circ} \mathrm{C}$ for $24 \mathrm{~h}$ on the farrowing rates and prolificacy after NsDU ET (Table 5). The results from this experiment demonstrated, for the first time, that high reproductive performance can be achieved after NsDU transfers of fresh embryos cultured for at least $24 \mathrm{~h}$ in a defined medium. 
Table 4 Survival rates and final developmental stages of porcine morulae/early blastocysts cultured for $24 \mathrm{~h}$ in different media and temperatures (means \pm SEM).

\begin{tabular}{lccccc}
\hline & \multicolumn{5}{c}{ Culture media } \\
\cline { 2 - 6 } & \multicolumn{2}{c}{ TL-HEPES-PVA } & \multicolumn{2}{c}{ NCSU-HEPES-BSA } & Control* \\
\hline Temperature $\left({ }^{\circ} \mathrm{C}\right)$ & 25 & 37 & 25 & 37 & 38.5 \\
No. of embryos & 59 & 57 & 58 & 58 & 31 \\
Embryo stage $(0 \mathrm{~h})^{* *}$ & $1.4 \pm 0.1$ & $1.4 \pm 0.1$ & $1.4 \pm 0.1$ & $1.4 \pm 0.1$ & $1.5 \pm 0.1$ \\
Survival rate $(\%)$ & $54.2 \pm 4.5^{\mathrm{a}}$ & $89.5 \pm 4.5^{\mathrm{b}}$ & $82.8 \pm 4.5^{\mathrm{b}}$ & $96.6 \pm 4.5^{\mathrm{b}}$ & $100 \pm 6.1^{\mathrm{b}}$ \\
Embryo stage $(24 \mathrm{~h})$ & $2.6 \pm 0.1^{\mathrm{a}}$ & $3.1 \pm 0.1^{\mathrm{b}}$ & $2.5 \pm 0.1^{\mathrm{a}}$ & $3.1 \pm 0.1^{\mathrm{b}}$ & $4.3 \pm 0.1^{\mathrm{c}}$ \\
\hline
\end{tabular}

*NCSU23-BSA-fetal calf serum medium at $38.5^{\circ} \mathrm{C}$ in an atmosphere of $5 \% \mathrm{CO}_{2}$ in air.

**The embryo stage at 0 and 24 h of culture was scored as follow: 1, morula; 2, early blastocyst; 3, full blastocyst; 4, expanded blastocyst; 5, hatching or hatched blastocyst. a,b,c, Means with different superscripts within the same row differ $(P<0.05)$.

Table 5 In vivo development of the porcine blastocysts derived from morulae/early blastocysts cultured for 24 $h$ in TL-HEPES-PVA medium at $37^{\circ} \mathrm{C}$ after the non-surgical deep-uterine transfers of 30 embryos to each of the asynchronous $(+24 \mathrm{~h})$ recipients.*

\begin{tabular}{lcc}
\hline & \multicolumn{2}{c}{ Culture period $(\mathrm{h})$} \\
\cline { 2 - 3 } & $0 \mathrm{~h}($ control)** & $24 \mathrm{~h} * * *$ \\
\hline No. of recipients & 18 & 19 \\
Pregnancy rate $(\mathrm{n} / \%)$ & $17(94.4)$ & $17(89.5)$ \\
Farrowing rate $(\mathrm{n} / \%)$ & $16(88.9)$ & $8.7 \pm 0.7$ \\
Total born (mean \pm SEM) & $9.3 \pm 0.9$ & $8.3 \pm 0.8$ \\
Born alive (mean \pm SEM) & $8.8 \pm 0.7$ & $8.5)$ \\
\hline
\end{tabular}

*TL-HEPES medium at $37{ }^{\circ} \mathrm{C}$ was used for collection, handling, culture, and transfers.

$* *$ Full blastocysts were collected and transferred to recipients within $3 \mathrm{~h}$ of collection.

$* * *$ Morulae/early blastocysts were cultured for $24 \mathrm{~h}$, and the resultant blastocysts were transferred to the recipients.

\section{Non-surgical deep-intrauterine transfer with vitrified/warmed embryos}

In addition to the applications of the ET technology, the development of a procedure to cryopreserve pig embryos would provide additional transcendental applications for the pig industry, which have been reviewed in detail (Dobrinsky 2001). Currently, vitrification is a uniquely suitable method for the long-term storage of porcine embryos. Since the development of the porcine open pulled straw (OPS) vitrification technology (Vajta et al. 1997), numerous factors have been studied to improve the survival of the vitrified/warmed embryos. These factors include the stage of embryonic development, the embryo pre-treatments (e.g., delipidation, cytoskeletal stabilization, and centrifugation), the vitrification containers, the cooling and warming rates, the cryoprotectant toxicity and concentration, and the composition of the vitrification and warming solutions (reviewed in Dobrinsky 2001, Berthelot et al. 2003, Martinez et al. 2005, Cameron et al. 2006).

From a practical standpoint, two important aspects should be emphasized. First, for hygienic reasons, the transportation is restricted to embryos with an intact zona pellucida. Therefore, the most appropriate stages for commercial ET are the morula and unhatched blastocyst. Second, cryopreservation protocols requiring no special embryo pre-treatments are the most suitable for commercial purposes. Post-warming, in vitro survival rates of $40-70 \%$ were initially reported with untreated morulae and blastocysts vitrified using the OPS method (reviewed in Berthelot 
et al. 2003, Cameron et al. 2006). Our laboratory and others have since obtained improved survival rates using untreated vitrified embryos (Table 6) so that similar survival rates have been reported for these embryos and their fresh counterparts (Sanchez-Osorio et al. 2010a). This finding confirms the results of another study indicating that the embryos require no pre-treatment prior to vitrification (Cuello et al. 2010).

Table 6 Reports concerning post-warming in vitro survival of untreated porcine embryos prior to vitrification since 2007.

\begin{tabular}{lcccl}
\hline Embryonic stage & Method* & Embryos vitrified (n) & Survival (\%) & Reference \\
\hline Morula & SOPS & 91 & 79.1 & Sanchez-Osorio et al. 2008 \\
& MMV & 40 & 55.0 & Fujino et al. 2008 \\
Unhatched & SOPS & 37 & 80.0 & Sanchez-Osorio et al. 2010a \\
Blastocyst & OPS & 128 & 67.0 & Berthelot et al. 2007 \\
& SOPS & 115 & 81.7 & Sanchez-Osorio et al. 2008 \\
& MMV & 32 & 84.4 & Fujino et al. 2008 \\
& MVC & 82 & 70.7 & Sakagami et al. 2010 \\
PFV & 81 & 79.0 & Sakagami et al. 2010 \\
& SOPS & 61 & 96.8 & Cuello et al. 2008 \\
& SOPS & 44 & 86.4 & Cuello et al. 2010 \\
& SOPS & 27 & 98.4 & Sanchez-Osorio et al. 2010a \\
& OPS/SOPS & 797 & 92.0 & Sanchez-Osorio et al. 2010b \\
SSV & 47 & 98.0 & Beebe et al. 2011 \\
SOPS & 60 & 93.3 & Gomis et al. 2012 \\
\hline
\end{tabular}

*OPS: open pulled straw; SOPS: super-fine OPS; MMV: metal mesh vitrification; MVC: minimum volume cooling; PVF: pullulan film vitrification; SSV: solid surface vitrification

Despite the advances achieved in the vitrification protocols, few reports on the in vivo survival after the surgical transfer of vitrified untreated morulae and blastocysts have appeared in last decade (Table 7). Although results from our laboratory indicate that these embryos are able to survive in vivo and yield acceptable farrowing rates (75\%) and litter sizes (9.9 piglets) when surgically transferred to the recipients, the further development of NsDU ET in combination with embryo vitrification could allow the widespread use of both technologies in the pig industry.

From a technical perspective, our laboratory has recently improved both methodologies. We demonstrated that morulae and blastocysts can be vitrified and warmed via SOPS in a chemically defined medium with no adverse effects on the in vitro survival (Sanchez-Osorio et al. 2010a). We have also simplified the conventional three-step warming procedure and developed a direct warming procedure (one-step dilution) for morulae and blastocysts vitrified via the OPS (Cuello et al. 2004a, Sanchez-Osorio et al. 2008) and SOPS (Gomis et al. 2012) methods. An acceptable farrowing rate $(42.9 \%)$ and litter size (5.4 piglets) were achieved when the one-step warming was combined with the OPS vitrification method and NsDU ET (Cuello et al. 2005). Recent modifications to the one-step warming method have yielded a promising farrowing rate $(50 \%)$ and litter size (10.4 piglets) after the NsDU ET of SOPS-vitrified morulae and blastocysts (Gomis et al. 2012). The results provided herein confirm that, despite the improvements over the past decade, more research is needed to increase the reproductive performance of the recipients when vitrification and NsDU ET are combined. 
Table 7 Reproductive performance of the recipients after surgical transfer of vitrified porcine blastocysts.

\begin{tabular}{ccccl}
\hline $\begin{array}{c}\text { Recipients } \\
(\mathrm{n})\end{array}$ & $\begin{array}{c}\text { Embryos } \\
\text { transferred }(\mathrm{n})\end{array}$ & $\begin{array}{c}\text { Pregnancy/ } \\
\text { farrowing }(\%)\end{array}$ & $\begin{array}{c}\text { Fetuses/ } \\
\text { piglets born }\end{array}$ & Reference \\
\hline 5 & $30-36$ & $100 / 40$ & 8.5 & Misumi et al. 2003 \\
20 & 20 & $80^{*}$ & $6.0^{*}$ & Cuello et al. 2004b \\
20 & $25-30$ & $80 / 75$ & 8.2 & Cameron et al. 2004 \\
7 & $28-30$ & $71.4^{*}$ & $7.2^{*}$ & Beebe et al. 2005 \\
40 & $20-30$ & $57.3^{*}$ & $8.3^{*}$ & Berthelot et al. 2007 \\
6 & $23-27$ & $83.3 / 83.3$ & 5.2 & Beebe et al. 2011 \\
3 & $21-26$ & $100 / 100$ & 9.6 & Matsunari et al. 2012 \\
48 & $20-30$ & $83.3 / 75.0$ & 9.9 & EA Martinez, C Cuello, MA Gil \& J Roca, \\
& & & & unpublished observations \\
\hline
\end{tabular}

*Data obtained at days 30-40 of pregnancy.

\section{Conclusion}

Non-surgical deep uterine ET is a simple, safe, effective and practical procedure that allows the commercial use of ET technology in the pig industry. Farrowing rates and litter sizes of $80 \%$ and 9.5 piglets, respectively, can be expected when using fresh morulae and blastocysts cultured for $0-6 \mathrm{~h}$ or fresh morulae/early blastocysts cultured for $24 \mathrm{~h}$. Although this culture time permits the international transport of embryos from the donor to the recipient farm, cryopreserved embryos are preferred because they can be stored indefinitely and used when necessary. Vitrification techniques continue to be improved. Currently, a high percentage (80-95\%) of untreated morulae and blastocysts survive the vitrification and warming procedures. High farrowing rates ( $75 \%$ ) and litter sizes (10 piglets) can also be obtained when the vitrified-warmed embryos are surgically transferred into the recipients. Unfortunately, when embryo vitrification and NsDU ET are combined, the farrowing rates are lower (50\%), although the litter sizes are maintained. Additional studies are currently being conducted on the factors affecting the in vivo survival after the NsDU transfer of vitrified embryos including the number of embryos transferred, the type of recipient (gilts or sows), and the administration of anti-prostaglandins to the recipients. The results from these and other studies will contribute to the widespread use of ET by the pig industry in the near future.

\section{Declaration of interest}

The authors declare that there is no conflict of interest that could be perceived as prejudicing the impartiality of the review reported.

\section{Funding}

Some of the studies presented in this review were supported in part by MINECO (AGL201238621), MICINN-FEDER (AGL2004-07546 and AGL2009-12091), MICINN (RYC2007-00651 and RYC2008-02081), SENECA (GERM 04543/07) and Formas, Stockholm, Sweden.

\section{Acknowledgments}

The authors are very grateful to Prof. Billy N. Day for his constant and fruitful help and invaluable advice over the years. We thank also the staff of the private companies Selección Batalle SA (Girona, Spain), Agropor SA (Murcia, Spain) and CEFUSA (Murcia, Spain) for the excellent management of the donors and recipients in the studies reported herein. 


\section{References}

Beebe LFS, Cameron RDA, Blackshaw AW \& Keates HL 2005 Changes to porcine blastocyst vitrification methods and improved litter size after transfer. Theriogenology 64 879-890.

Beebe LFS, Bouwman EG, Mcllfatrick SM \& Nottle MB 2011 Piglets produced from in vivo blastocysts vitrified using the Cryologic Vitrification Method (solid surface vitrification) and a sealed storage container. Theriogenology 75 1453-1458.

Berthelot F, Martinat-Botte F, Vajta G \& Terqui M 2003 Cryopreservation of porcine embryos: state of the art. Livestock Production Science 83 73-83.

Berthelot F, Venturi E, Cogné J, Furstoss V \& MartinatBotte F 2007 Development of OPS vitrified pig blastocysts: Effects of size of the collected blastocysts, cryoprotectant concentration used for vitrification and number of blastocysts transferred. Theriogenology 68 178-185.

Blum-Reckow B \& Holtz W 1991 Transfer of porcine embryos after 3 days of in vitro culture. Journal of Animal Science 69 3335-3342.

Brüssow KP, Schneider F, Kanitz W, Rátky J, Kauffold J \& Wähner M 2009 Studies on fixed-time ovulation induction in the pig. Society of Reproduction and Fertility Supplement 66 187-195.

Caárdenas H \& Pope WF 2002 Control of ovulation rate in swine. Journal of Animal Science $\mathbf{8 0}$ (Supplement 1) E36-E46.

Cameron RDA, Durack M, Fogarty R, Putra DKH \& McVeigh J 1989 Practical experience with commercial embryo transfer in pigs. Australian Veterinary Journal 66 314-318.

Cameron RD, Beebe LF, Blackshaw AW \& Keates HL 2004 Farrowing rates and litter size following transfer of vitrified porcine embryos into a commercial swine herd. Theriogenology 61 1533-1543.

Cameron RD, Beebe LF \& Blackshaw AW 2006 Cryopreservation and transfer of pig embryos. Society of Reproduction and Fertility Supplement 62 277-291.

Cuello C, Gil MA, Parrilla I, Tornel J, Vazquez JM, Roca J, Berthelot F, Martinat-Botté F \& Martinez EA 2004a In vitro development following one-step dilution of OPS-vitrified porcine blastocysts. Theriogenology 62 1144-1152.

Cuello C, Berthelot F, Martinat-Botté F, Guillouet $P$, Furstoss V, Boisseau C, Manceau P, Locatelli A \& Martinez EA 2004b Transfer of vitrified blastocysts from one or two superovulated Large White Hyperprolific donors to Meishan recipients: reproductive parameters at day 30 of pregnancy. Theriogenology 61 843-850.

Cuello C, Berthelot F, Martinat-Botte F, Venturi E, Guillouet P, Vazquez JM, Roca J \& Martinez EA 2005 Piglets born after non-surgical deep intrauterine transfer of vitrified blastocysts in gilts. Animal Reproduction Science 85 275-286.

Cuello C, Gil MA, Almiñana C, Sanchez-Osorio J, Parrilla I, Caballero I, Vazquez, JM, Roca J, Rodriguez-
Martinez H \& Martinez EA 2007 Vitrification of in vitro cultured porcine two-to-four cell embryos. Theriogenology 68 258-264.

Cuello C, Sanchez-Osorio J, Almiñana C, Gil MA, Perals ML, Lucas X, Roca J, Vazquez JM \& Martinez EA 2008 Effect of the cryoprotectant concentration on the in vitro embryo development and cell proliferation of OPSvitrified porcine blastocysts. Cryobiology 56 189-194.

Cuello C, Sanchez-Osorio J, Almiñana C, Gil MA, Parrilla I, Roca J, Vazquez JM \& Martinez EA 2010 Superfine open pulled Straw vitrification of porcine blastocysts does not require pretreatment with cytochalasin B and/or centrifugation. Reproduction Fertility and Development 22 808-817.

De Winter PJJ, Verdonck M, De Kruif A, Coryn M, Deluyker HA, Devriese LA \& Haesebrouck F 1996 The relationship between the blood progesterone concentration at early metoestrus and uterine infection in the sow. Animal Reproduction Science 41 51-59.

Dobrinsky JR 2001 Cryopreservation of swine embryos: a chilly past with a vitrifying future. Theriogenology 56 1333-1344.

Ducro-Steverink DWB, Peters CGW, Maters CC, Hazeleger W \& Merks JWM 2004 Reproduction results and offspring performance after non-surgical embryo transfer in pigs. Theriogenology 62 522-531.

Fujino Y, Kojima T, Nakamura Y, Kobayashi H, Kikuchi K \& Funahashi H 2008 Metal mesh vitrification (MMV) method for cryopreservation of porcine embryos. Theriogenology 70 809-817.

Furstoss V, Berthelot F, Plat M, Venturi E, Royer E, Elleboudt F \& Martinat-Botté F 2012 Genetic and non-genetic parameters related to embryo production in superovulated Large White (LW) gilts. Animal Reproduction Science 134 177-183.

Guthrie HD, Henricks DM \& Handlin DL 1974 Plasma hormone levels and fertility in pigs induced to superovulate with PMSG. Journal of Reproduction and Fertility 41 361-370.

Gomis J, Cuello C, Sanchez-Osorio J, Gil MA, Parrilla I, Angel MA, Maside C, del Olmo D, Vazquez JM, Roca J \& Martinez EA 2012 Non-surgical deep intrauterine transfer of superfine open pulled straw (SOPS)-vitrified porcine embryos: evaluation of critical steps of the procedure. Theriogenology 78 1339-1349.

Hazeleger W 1999 Non-surgical embryo transfer in pigs. PhD thesis, University of Wageningen, The Netherlands.

Hazeleger W \& Kemp B 1994 Farrowing rate and litter size after transcervical embryo transfer in sows. Reproduction in Domestic Animals 29 481-487.

Hazeleger W \& Kemp B 2001 Recent developments in pig embryo transfer. Theriogenology 56 1321-1333.

Hazeleger W, Bouwman EG, Noordhuizen JPTM \& Kemp B 2000a Effect of superovulation induction on embryonic development on day 5 and subsequent development and survival after nonsurgical embryo transfer in pigs. Theriogenology 53 1063-1070. 
Hazeleger W, Noordhuizen JPTM \& Kemp B 2000b Effect of asynchronous non-surgical transfer of porcine embryos on pregnancy rate and embryonic survival. Livestock Production Science 64 281-284.

Holtz W \& Schlieper B 1991 Unsatisfactory results with the transfer of embryos from gilts superovulated with PMSG and hCG. Theriogenology 35 1237-1249.

James JE, Reeser PD, Davis DL, Straiton EC, Talbot AC \& Polge C 1980 Culture and long-distance shipment of swine embryos. Theriogenology 14 463-469.

Macháty Z, Day BN \& Prather RS 1998 Development of early porcine embryos in vitro and in vivo. Biology of Reproduction 59 451-455.

Martinez EA, Vazquez JM, Roca J, Lucas X, Gil MA \& Vazquez JL 2001 Deep intrauterine insemination and embryo transfer in pigs. Society of Reproduction and Fertility Supplement 58 301-311.

Martinez EA, Caamaño JN, Gil MA, Rieke A, Mccauley TC, Cantley TC, Vazquez JM, Roca J, Vazquez JL, Didion B, Murphy CN, Prather RS \& Day DN 2004 Successful nonsurgical deep uterine embryo transfer in pigs. Theriogenology 61 137-146.

Martinez EA, Vazquez JM, Roca J, Cuello C, Gil1 MA, Parrilla I \& Vazquez JL 2005 An update on reproductive technologies with potential short-term application in pig production. Reproduction in Domestic Animals 40 300-309.

Matsunari $H$, Maehara $M$, Nakano K, Ikezawa $Y$, Hagiwara Y, Sasayama N, Shirasu A, Ohta H, Takahashi M \& Nagashima H 2012 Hollow fiber vitrification: a novel method for vitrifying multiple embryos in a single device. Journal of Reproduction and Development 58 599-608.

Misumi K, Suzuki M, Sato S \& Saito N 2003 Successful production of piglets derived from vitrified morulae and early blastocysts using a microdroplet method. Theriogenology 60 253-260.

Niemann H, Wüst A \& Gardon JC 1989 Successful intercontinental transport of porcine embryos from Europe to South America. Theriogenology 31 525-530.
Polge C 1982 Embryo transplantation and preservation. In Control of Pig Reproduction pp 277-291 Eds DJA Cole and GR Foxcroft. Butterworth, London.

Polge C \& Day BN 1968 Pregnancy following non-surgical egg transfer in pigs. Veterinary Record $\mathbf{8 2} 712$.

Rubio Pomar FJ, Ducro-Steverink DWB, Hazeleger W, Teerds KJ, Colenbrander B \& Bevers MM 2004 Development, DNA fragmentation and cell death in porcine embryos after $24 \mathrm{~h}$ storage under different conditions. Theriogenology 61 147-158.

Sakagami N, Yamamoto T, Akiyama K, Nakazawa Y, Kojima N, Nishida K, Yokomizo S, Takagi Y, Abe H, Suzuki C \& Yoshioka K 2010 Viability of porcine embryos after vitrification using water-soluble pullulan films. Journal of Reproduction and Development $\mathbf{5 6}$ 279-284.

Sanchez-Osorio J, Cuello C, Gil MA, Almiñana C, Parrilla I, Caballero I, Garcia EM, Vazquez JM, Roca J \& Martinez EA 2008 Factors affecting the success rate of porcine embryo vitrification by the Open Pulled Straw method. Animal Reproduction Science $108334-344$.

Sanchez-Osorio J, Cuello C, Gil MA, Parrilla I, Maside C, Almiñana C, Lucas X, Roca J, Vazquez JM \& Martinez EA 2010a Vitrification and warming of in vivo-derived porcine embryos in a chemically defined medium. Theriogenology 73 300-308.

Sanchez-Osorio J, Cuello C, Gil MA, Parrilla I, Almiñana C, Caballero I, Roca J, Vazquez JM, RodriguezMartinez H \& Martinez EA 2010b In vitro post warming viability of vitrified porcine embryos: Effect of cryostorage length. Theriogenology 74 486-490.

Vajta G, Holm P, Greve T \& Callesen H 1997 Vitrification of porcine embryos using the open pulled straw (OPS) method. Acta Veterinaria Scandinavica 38 349-352.

Wallenhorst S \& Holtz W 1999 Transfer of pig embryos to different uterine sites. Journal of Animal Science 77 2327-2329.

Wilde MH, Xie S, Day ML \& Pope WF 1988 Survival of small and large littermate blastocysts in swine after synchronous and asynchronous transfer procedures. Therigenology 30 1069-1074. 